Instituto Internacional de Investigación y Desarrollo Tecnológico Educativo INDTEC, C.A.

DOI: https://doi.org/10.29394/scientific.issn.2542-2987.2017.2.3.17.310-324

OAl-PMH: http://www.indteca.com/ojs/index.php/Revista Scientific/oai

\title{
Plan de Trabajo Basado en la Implementación de la Técnica del Huerto del Pie Cuadrado como Alternativa para el Aprovechamiento de los Desechos Sólidos Orgánicos
}

(Caso de Estudio: Habitantes del Barrio el Paraíso Sector San Rafael de la Parroquia Barinitas, Municipio Bolívar, Estado Barinas)

Autora: Sandra Yolimar Delgado Briceño Universidad Pedagógica Experimental Libertador, UPEL sdelgado0912@hotmail.com

Barinas, Venezuela

\section{Resumen}

La presente investigación tiene como propósito Promover un plan de trabajo basado en la implementación de la técnica del huerto del pie cuadrado como alternativa para el aprovechamiento de los desechos sólidos orgánicos en la comunidad del Barrio El Paraíso del Sector San Rafael de la Parroquia Barinitas Municipio Bolívar Estado Barinas durante el Período 2014-2015. El estudio se enmarcó en el paradigma cualitativo bajo el método de la investigación acción participante. La técnica utilizada para la recolección de la información fue la observación participante y la entrevista, aplicando un registro audiovisual cara a cara y como instrumento la guía de entrevista, con un diseño de investigación de campo. Los informantes claves lo conformarán tres habitantes de la comunidad. La autenticidad y viabilidad de los instrumentos aplicados se hizo a través de la triangulación, en cuanto a la técnica de análisis de información se elaboró el cuadro de categorías con el cual se realizaron comparaciones y posibles contrastes. Con la promoción de este plan se pretende disminuir de forma definitiva la acumulación de desechos sólidos orgánicos, mejorar la calidad de vida de los habitantes del sector antes mencionado, conservando y preservando el ambiente que los rodea.

Palabras clave: plan de trabajo; huerto de pie cuadrado; desechos sólidos orgánicos.

Fecha de Recepción: 14-09-2016

Fecha de Aceptación: 20-10-2016 


\author{
Work Plan Based on the Implementation of the Technical Square Foot \\ Gardening as an Alternative to the Use of Solid Waste Organics \\ (Case Study: Residents of Barrio San Rafael Paradise Sector of Barinitas \\ Parish, Bolivar County, State Barinas)
}

\begin{abstract}
This research aims to promote a work plan based on the implementation of the technique of Square Foot Gardening as an alternative to the use of organic solid waste in the community of Barrio El Paraiso del Sector San Rafael de la Barinitas Parish Bolivar County Barinas State during the period 20142015. The study was part of the qualitative paradigm under the method of participatory action research. The technique used for collecting information was participant observation and interview, using an audiovisual record face to face and as an instrument interview guide, with a design of field research. Key informants make up the three residents of the community. The authenticity and viability of the instrument was applied through triangulation, in regard to technical information analysis table of categories with which comparisons and contrasts were made possible was developed. With the promotion of this plan it is to permanently reduce the accumulation of organic solid waste, improve the quality of life of the inhabitants of the aforementioned sectors, conserving and preserving the environment around them.
\end{abstract}

Keywords: work plan; square foot gardening; organic solid waste.

Date Received: 14-09-2016

Date Acceptance: $20-10-2016$ 


\section{Introducción}

La acumulación de los desechos sólidos es un problema a nivel mundial, que ha venido afectando de forma directa al ambiente y a la salud pública. El principal protagonista de estas acciones ha sido la mano del hombre, quien bajo las condiciones de consumismo y en búsqueda de una mejor calidad de vida ha hecho uso irracional de los recursos de la madre naturaleza, contaminando todo a su paso sin importar las consecuencias que produce tanto al planeta como así mismo. Todo esto se ha transformado en el transcurrir de los años en grandes focos de contaminación que afectan los suelos, el aire, el agua, la fauna y la flora, de tal forma, que en cada espacio de acumulación de desechos sólidos existirá la presencia de animales roedores, insectos como el mosquito, la mosca y que son propagadores de enfermedades y epidemias que afectan a la salud.

La basura es un problema de todos los días y se ha convertido en una gran fuente de contaminación ambiental, malos olores y microorganismos patógenos, trayendo como consecuencia enfermedades catastróficas para el hombre. En la actualidad muchas comunidades del mundo han creído que la basura no constituye un problema que pueda afectar profundamente la existencia de los seres vivos en el planeta. Están tan preocupados por otros problemas de tipo económico, político y social que no le han prestado la debida atención a la contaminación del ambiente y de la salud pública producida por la basura y la mala disposición de la misma.

Los desechos sólidos son producidos por la mano del hombre y pueden ser generados desde los hogares mediante desperdicios tales como los restos de comida, poda de árboles, maleza, plantas ornamentales, materiales que ya no son de utilidad como el papel, el cartón, el vidrio, el plástico entre otros. Todos estos residuos sólidos, en su mayoría son susceptibles de reaprovecharse o transformarse con un correcto reciclado.

Por consiguiente, la basura o residuos sólidos es la respuesta a lo que 
se consume, se compra, se desperdicia, y a las relaciones sociales que giran en torno a estos fenómenos. Ahora bien, la basura dispuesta de una forma inadecuada puede traer consecuencias letales y es preocupante observar como los desechos sólidos van incrementándose en el transcurrir de los años. La sobrepoblación mundial, el consumismo y la falta de cultura ambiental son indicadores de una incontrolable acción hacia la contaminación y generación de desechos, lo que traerá como consecuencia una crisis ambiental y de salud de la cual no se podrá escapar ni con los mejores proyectos del mundo.

De igual forma, en Venezuela la organización no gubernamental, Fundación Vitalis (2000), expresa su preocupación en cuanto a que:

De 18 mil toneladas de residuos que se producen diariamente en el país, pudieran estarse reciclando solamente de 15 a $20 \%$ del total de los residuos. De acuerdo con las estadísticas que maneja esta organización, en Venezuela se recicla alrededor del $95 \%$ del aluminio, $90 \%$ de hierro, $25 \%$ de vidrio, $1 \%$ de materia orgánica, $20 \%$ de papel y cartón, y alrededor del $2 \%$ en plásticos. Sin embargo, en función del volumen total de residuos, menos de la quinta parte pudieran estar recibiendo un tratamiento final apropiado.

Se puede apreciar en estos porcentajes la poca cantidad de residuos sólidos que han estado recibiendo un tratamiento adecuado, lo que indica que los niveles de contaminación producto de la generación de residuos podrían incrementarse aún más en los años venideros.

Por ello, es oportuno mencionar la elaboración de planes enmarcados a abordar la problemática ambiental generada por los desechos sólidos orgánicos. Tal es el caso de la técnica del huerto del pie cuadrado, que consiste en un sistema de producción en camas de cultivo que esta reticulado o dividido en cuadrados de 1 pie $\times 1$ pie (aprox. $30 \mathrm{~cm} \times 30 \mathrm{~cm}$ ), donde se puede plantar o sembrar cultivos o especies diferentes en cada cuadrado, utilizando sustrato altamente nutritivo, que mantenga una buena humedad, sea aireado, 
no muy suelto y que sea apto para todo tipo de plantas. Mediante esta técnica se puede abordar la problemática de acumulación de los desechos sólidos orgánicos, implementando un plan que permita darle uso adecuado a tales desechos, aprovechándolos para la elaboración de abono orgánico que pueden ser utilizados en la siembra a través la técnica del huerto del pie cuadrado. De igual forma, la ejecución del plan en base a esta técnica contribuiría a mejorar y conservar el medio ambiente.

En función de lo antes expuesto surgen las siguientes interrogantes:

¿Cuál es el uso de la técnica del huerto del pie cuadrado por los habitantes de la comunidad del Barrio El Paraíso del Sector San Rafael?

¿Cómo sería el plan de trabajo basado en la aplicación de la técnica del huerto del pie cuadrado como alternativa para el aprovechamiento de los desechos sólidos orgánicos en los habitantes de la comunidad del Barrio El Paraíso del Sector San Rafael?

¿Cómo se ejecutará el plan de trabajo basado en la técnica del huerto del pie cuadrado como alternativa para el aprovechamiento de los desechos sólidos orgánicos en los habitantes de la comunidad del Barrio El Paraíso del Sector San Rafael?

¿Cómo sería la evaluación del impacto social, ambiental y educativo de la ejecución del plan de trabajo basado en la técnica del huerto del pie cuadrado para el aprovechamiento de los desechos sólidos orgánicos en la comunidad del Barrio El Paraíso del Sector San Rafael? 


\section{Plan de Trabajo}

Un plan de trabajo es un instrumento de planificación, para lo cual lo define Álvarez (2012), como:

Un esquema de un conjunto de metas y procesos mediante los cuales un equipo o una persona puede lograr esos objetivos, ofreciendo al lector una mejor comprensión del enfoque del proyecto. Los planes de trabajo, tanto si se utilizan en la vida profesional o la académica, ayudan a mantener la organización mientras se trabaja en algún proyecto. A través de los planes de trabajo, uno divide un proceso en tareas pequeñas y alcanzables e identifica las cosas que se quieren lograr.

En función de esto, el plan de trabajo es un instrumento a manera de esquema que sirve para dividir los procesos de una meta, en actividades pequeñas y que son factibles de lograrse. Pueden ser utilizados por profesionales o bien por estudiantes, mediante el cual ellos esquematizan las metas y los procesos que se pretenden alcanzar.

\subsection{Desechos Sólidos}

Uno de los mayores problemas ambientales de Venezuela son los desechos sólidos, los cuales son generados por las actividades humanas y que afectan gravemente al ambiente a la salud. En este sentido, Hernández (1990), define los desechos sólidos cómo:

Todos aquellos residuos que provienen de las actividades humanas y/o de animales generalmente sólidos, y que se consideran inútiles (relativamente) e indeseables. En la realidad los desechos sólidos resultan de las diversas actividades desarrolladas por el hombre, por más simples que están seas, en su afán de mejorar la calidad de vida de la especie humana (pág. 153).

En la definición que postula este autor se entiende claramente que los desechos sólidos son el resultado de la actividad humana en busca de mejorar su calidad de vida. 


\subsection{La Horticultura Por Pie Cuadrado}

En una técnica de siembra muy útil que puede aplicarse en espacios relativamente pequeños. Según Javi (2012), explica claramente que es una técnica que:

Fue desarrollada por Mel Bartholomew a finales de la década de 1970 con la meta de producir una mayor cosecha en menos espacio y con menos trabajo. Usando estos métodos de horticultura cada cuadrado de un pie por un pie puede ser sembrado con una hortaliza, hierba o flor diferente, o, si quiere una gran cantidad de algo en particular puede plantar lo mismo en varios cuadrados. Fácilmente puede plantar, despejar, y replantar un cuadrado durante la estación sin alterar otras áreas de su huerto. Cada cuadrado puede ser manejado simple e independientemente.

De acuerdo a este artículo, se puede decir que el huerto de pie cuadrado es una técnica que permite contar con varios cultivos en un espacio de un metro cuadrado; es decir, con medidas exactas de un pie por un pie. Este espacio cuadrado se puede implementar tanto en espacios grandes como en los pequeños. Al parecer este método es muy efectivo y tiene múltiples seguidores en países desarrollados, se consiguen buenas producciones y se evitan los trabajos que requiere plantar en tierra, que son lo más duros en horticultura y jardinería. Se puede cultivar cualquier cosa así desde hortalizas, tubérculos, verduras, hasta todo tipo de flores y hierbas aromáticas.

\subsection{Perspectivas Legales}

En las perspectivas legales se señalaron los artículos 107, 127 y 129 de la Constitución de la República Bolivariana de Venezuela (2000): los cuales hacen referencia al derecho a la educación ambiental, a los deberes y derechos de gozar de un ambiente sano y al cuidado y conservación del mismo. De igual forma se citarón los artículos 54,55 y 58 de la Ley de los Residuos y Desechos Sólidos (2004): en la cual hacen énfasis en el adecuado 
tratamiento de los desechos sólidos orgánicos y de lo perjudicial que pueden ser para la salud. En este mismo orden de ideas, hay que resaltar el artículo 2 de la Ley Orgánica de Los Consejos Comunales, que hacen referencia a que las comunidades son instancias de participación en pro de la búsqueda de sus propias necesidades y problemas, así como también la solución común de los mismos.

\section{Naturaleza de la Investigación}

Esta investigación por su condición reviste un carácter Cualitativo para lo cual Bonilla y Rodríguez (1997), la conceptualiza como:

La Investigación Cualitativa intenta hacer una aproximación global de las situaciones sociales para explorarlas, describirlas y comprenderlas de manera inductiva... a partir de los conocimientos que tienen las diferentes personas involucradas en ella... esto supone que los individuos interactúan (pág. 70).

En atención a lo planteado por este autor, en la investigación cualitativa se busca comprender una situación o problema mediante el estudio exploratorio, lo que permitirá describir y comprender los procesos involucrados en el objeto de estudio partiendo de lo que conocen las personas que están inmersas en la situación problema.

\subsection{Tipo de Investigación}

El tipo de investigación es la investigación acción participante que según, Basagoiti (2001), refiere que:

Es una metodología que permite desarrollar a los investigadores un análisis participativo, donde los actores implicados se convierten en los protagonistas del proceso de construcción del conocimiento de la realidad sobre el objeto de estudio, en la detección de problemas y necesidades, en la elaboración de propuestas y soluciones, es decir la transformación social (pág. 75). 
En esta investigación los actores son los habitantes de la comunidad quienes van a interactuar en los procesos de solución al problema planteado, participando directamente en la ejecución del plan de acción adecuado a sus necesidades y bajo los recursos disponibles al alcance de sus manos sin que incurra en gastos mayores, pero aportando soluciones efectivas y significativas.

\subsection{Diseño de la Investigación}

El estudio se ubica como una investigación de campo. Según la Universidad Pedagógica Experimental Libertador (2007), la investigación de campo, es aquella que se refiere a:

Análisis sistemático de problemas en la realidad, con el propósito bien sea de describirlos, interpretarlos, entender su naturaleza y factores constituyentes, explicar sus causas y efectos, o predecir su ocurrencia, haciendo uso de métodos característicos de cualquiera de los paradigmas o enfoques conocidos o en desarrollo (pág. 14).

De acuerdo con esto, el diseño se adecua a esta investigación ya que permite describir la problemática planteada, interpretando las causas y consecuencias que lo producen utilizando como modelo la metodología cualitativa. Además, los datos serán extraídos en forma directa de la realidad y por la propia investigadora, a través del uso de instrumentos para recolectar la información relacionada con la acumulación de los desechos sólidos orgánicos que se acumulan es las casa y el por qué realizan estas prácticas, así como también estudiar las posibles consecuencias que esto pudiera estar trayendo a los habitantes de la comunidad.

\subsection{Informantes Claves}

Los sujetos que suministraron la información en el presente estudio son los informantes claves que según Mckernan (1996), quien define los 
informantes claves de la siguiente manera:

Los informantes claves se escogen porque tiene tiempo y conocimiento especial para proporcionar relatos y comentarios determinados y perspicaces que a menudo los diseños distribuidos al azar niegan al investigador. Es prudente e instructivo advertir que los informantes claves a menudo son atípicos y tiene que elegirse con cuidado para asegurar la representatividad. Los informantes claves están enterados de las cuestiones y tareas por su experiencia y fondo cultural. Debido que están en posiciones clave, a menudo tiene una elevada educación y destreza de reflexión que pueden producir hipótesis y líneas adicionales de indagación para el investigador (pág. 152).

Los sujetos que suministraron la información del estudio se seleccionaron de manera intencional. Estos informantes claves son personas con un alto grado de solvencia moral, con una gran educación y con una edad que les permite expresarse de forma reflexiva, crítica e incluso creativa, capaz de aportar opiniones claras y que sirvieron de ayuda al investigador. También fueron escogidos porque conocen de cerca la problemática objeto de estudio. En este sentido, se seleccionaron como informantes tres habitantes de la comunidad quienes dieron testimonio sobre el uso de la técnica del huerto del pie cuadrado en sus hogares.

\subsection{Técnicas e Instrumentos de Recolección de Información}

En esta investigación se aplicó la observación participante para lo cual DeMunck y Sobo (1998): describen "la observación participante como el primer método usado por los antropólogos al hacer trabajo de campo. El trabajo de campo involucra "mirada activa, una memoria cada vez mejor, entrevistas informales, escribir notas de campo detalladas, y, tal vez lo más importante, paciencia" (pág. 148).

Por otra parte, se puede mencionar, que en esta investigación se utilizó para la recolección de los datos la técnica de la entrevista y el registro 
audiovisual, todo esto como proceso fundamental para el desarrollo de la investigación

Según Arias (1999): "los instrumentos son los medios materiales que se emplean para recoger y almacenar la información" (pág. 53). Para recoger datos e información relevantes, la investigadora utilizó como instrumentos de recolección de datos el guión de entrevista para lo cual, Valles (1997), lo define de la siguiente manera:

No existe entrevista en profundidad que prescinda de un guión, una orientación de preguntas, temas o subtemas cuyo orden no es cerrado ni debe seguirse de forma rígida y su empleo hace posible la captación de aspectos que no están referidos en él y perfectamente pueden incorporarse (pág. 197).

En función de esta definición, en esta investigación se aplicó el guión de entrevista ya que para el momento de la investigación este instrumento iba a permitir obtener información sobre la situación problema con preguntas abiertas. Este instrumento permitió incluir otras preguntas que no se habían formulado en el momento de la aplicación del instrumento.

\subsection{Autenticidad y Fiabilidad de la Información}

La autenticidad y fiabilidad son cualidades esenciales que deben estar presentes en todos los instrumentos de carácter científico para la recogida de datos, para lo cual, Pérez (1998), acota que: "si el instrumento o instrumentos reúnen estos requisitos habrá cierta garantía de los resultados obtenidos en un determinado estudio y, por lo tanto, las conclusiones pueden ser creíbles y merecedoras de una mayor confianza" (pág. 71).

Por otra parte, Hernández y otros (1998), sostienen que:

En la investigación cualitativa no calculamos un coeficiente o índice de confiabilidad, ni medidas estadísticas de validez, más bien analizamos en profundidad (producto de la reflexión) aspectos tales como: si los sujetos son sinceros o abiertos, si se registran los sucesos 0 acontecimientos relevantes al 
problema de investigación, si se recolectó la información necesaria y suficiente para el cumplimiento de los objetivos del estudio, si las preguntas que se realizarán fueron pertinente o adecuadas, entre otros aspectos (pág. 475).

De acuerdo a este autor, se puede acotar que en la investigación cualitativa no hay fórmula que permita calcular la autenticidad y fiabilidad del instrumento, sino que las mismas se obtendrán de un conjunto de elementos tales como la opinión de los sujetos, de los registros de los sucesos y de otros aspectos relevantes que brindarán validez a la investigación como tal.

En esta investigación la autenticidad y fiabilidad del instrumento se realizó mediante la triangulación de datos. De esta forma, se pudo realizar comparaciones entre las opiniones de los sujetos informantes.

\section{Conclusiones}

Con el plan de trabajo basado en la implementación de la técnica del huerto del pie cuadrado como alternativa para el aprovechamiento de los desechos sólidos orgánicos se logró erradicar la quema de desechos sólidos orgánicos en los patios de las viviendas de los habitantes de la comunidad del Barrio El Paraíso del Sector San Rafael. De igual forma, lograron adquirir aprendizaje en cuanto a la elaboración de compostero para producir abono orgánico a partir de los desechos sólidos orgánicos y con ellos sembraron varios rubros agrícolas aplicando la técnica de huerto de pie cuadrado. En conjunto se logró concienciar a la comunidad en general para que cuidaran y conservaran el ambiente. Contribuyeron a no quemar, por lo que ahora se puede disfrutar de un aire puro y actualmente se conservan más las propiedades de los suelos para proyectos socioproductivos a futuro.

\section{Referencias}

Arias, G. (1999). El Proyecto de Investigación Guía para su Elaboración. 
Caracas: Editorial Episteme C.A.

Álvarez, O. (2012). Cómo crear un Plan de Trabajo. Wikihow. [Documento en línea]. Recuperado de: http://es.wikihow.com/crear-un-plan-detrabajo/

Constitución de la República Bolivariana de Venezuela (2000). Gaceta Oficial de la República Bolivariana de Venezuela, 5.453, de fecha 24 de marzo. Caracas, Venezuela: Asamblea Nacional Constituyente.

Basagoiti, R. (2001). La Investigación Acción Participante de bolsillo. Madrid: Edita ACSUR.

Bonilla, C., y Rodríguez, S. (1997). Más allá del dilema de los métodos. La investigación en ciencias sociales. 3era edición. Santafé de Bogotá. Ediciones Uniandes.

DeMunck, V., \& Sobo, E. (Eds.) (1998). Using Methods in the Field: A Practical Introduction and Casebook. Walnut Creek, CA: AltaMira Press.

Fundación Vitalis (2000). Basura: Destino Incierto en Venezuela. [Página web en línea]. Recuperado de: http://www.vitalis.net/recursos/residuosy-desechos/reciclaje/

Hernández, y otros (1998). Metodología de la Investigación. México: McGraw-Hill / Interamericana Editores, S.A. de C.V.

Hernández, P. (1990). Contaminación Ambiental. Maracaibo, Venezuela. Javi (2012). Guía de Jardinería. [Documento en línea]. Recuperado de: http://www.guiadejardineria.com/el-metodo-del-pie-cuadrado/ Ley de Residuos y Desechos Sólidos (2004). Gaceta Oficial de La República Bolivariana de Venezuela, 38.068, noviembre 18. Venezuela.

Mckernan, J. (1996). Investigación-acción y curriculum: Métodos y recursos para profesionales reflexivos. ISBN: 84-7112-438-6. España: Ediciones Morata, S.L.

Pérez, G. (1998). Investigación Cualitativa. Retos e interrogante. Tomo I y 


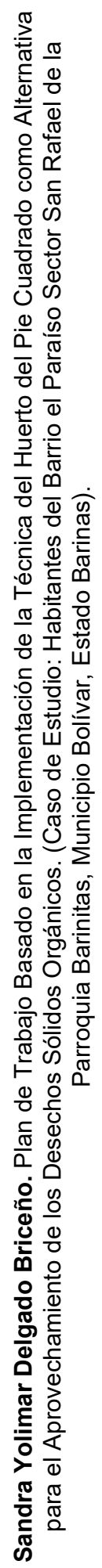

II. España: Editorial La Muralla.

Universidad Pedagógica Experimental Libertador (2007). Manual de Trabajos de Grado de Especialización y Maestría y Tesis Doctorales. Caracas: FEDUPEL.

Valles, M. (1997). Técnicas cualitativas de investigación social. Reflexión metodológica y práctica profesional. Madrid: Síntesis. 


\section{Sandra Yolimar Delgado Briceño \\ e-mail: sdelgado0912@hotmail.com}

Inicié mi carrera profesional en el Tecnológico "Antonio

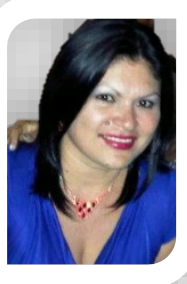

José de Sucre" en donde obtuve el título de T.S.U. en

Informática en el año 2001. Ingrese en ese mismo año al

Ministerio de Educación como docente de aula en el Liceo

Bolivariano "Cándido Antonio Meza". Continué mi

formación profesional y me gradué con honores en el

Tecnológico "Agustín Codazzi” en la carrera de Educación Integral. En el año 2008 obtuve el título de Licda. En Educación Integral en la Universidad "Simón Rodríguez". En el 2015 culmine la escolaridad de postgrado en la carrera de Educación, ambiente y desarrollo en la UPEL.

El contenido de este manuscrito se difunde bajo una Licencia de Creative Commons ReconocimientoNoComercial-Compartirlgual 4.0 Internacional 